\title{
The profile of health care utilization among HIV/AIDS patients in Iran from 1987 to 2016: A nationwide study
}

\author{
Zahra Mirmoghadam $^{\mathrm{a}}$, Manoochehr Karami ${ }^{\mathrm{a}, \mathrm{b}}$, Younes Mohammadi ${ }^{\mathrm{a}, \mathrm{c}, *}$, Mohammad Mirzaei $^{\mathrm{a}}$

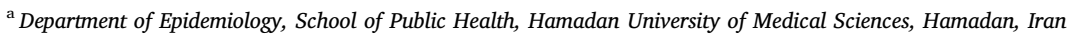 \\ ${ }^{\mathrm{b}}$ Modeling of Noncommunicable Diseases Research Center, Department of Biostatistics and Epidemiology, School of Public Health, Hamadan University of Medical \\ Sciences, Hamadan, Iran \\ ${ }^{\mathrm{c}}$ Social Determinants of Health Research Center, School of Public Health, Hamadan University of Medical Sciences, Hamadan, Iran
}

A R T I C L E IN F O

\section{Keywords:}

HIV/AIDS

Health care

Utilization

Iran

\begin{abstract}
A B S T R A C T
Background: The Acquired Immunodeficiency Syndrome (AIDS) as a major threat to public health is the most important infectious pandemic of the late 20th and the early 21st centuries across the globe. This study aimed to assess the status of health service utilization provided by the Iranian Ministry of Health and Medical Education (MOHME) for people living with HIV/AIDS (PLWHA) in Iran from 1987 to 2016.

Methods: Samples were 30516 patients with HIV/AIDS from 31 Iranian provinces registered in the Iranian health database from 1987 to October 2016. Health care services provided by the MOHME were assessed in terms of age, gender, and the marital status. Descriptive and inferential statistics were used for data analysis and the significance level was set as $\mathrm{P}<0.05$.

Results: Out of 30516 patients with HIV/AIDS in the age group of $35-44$ years, $83.58 \%(n=25506)$ were male and $41.52 \%(n=12670)$. The highest coverage of health care services provided to these patients included the laboratory test of the cluster of differentiation 4 cells count (CD4), the latest status of tuberculosis (TB), Purified Protein Derivative (PPD) diagnostic test, and treatment using Antiretroviral drug (ARV). However, the coverage other health care services was very low. We found that there was a statistically significant association between gender, age and the marital status, and receiving health care services. There was a strong correlation between the gender of a woman and cut the last TB regime (OR: $2.54 \mathrm{P}<0.001$ ).

Conclusions: While the process of providing health care services to such patients has improved in recent years, it is far from the expected level and the overall coverage is very low. Planning for the appropriate supply and distribution of Health Care Workers (HCWs) and specialized facilities, provision of drugs, continuous education of HCWs, improvement of registration and reporting of HIV/AIDS can enhance the provision of healthcare services to these patients.
\end{abstract}

\section{Introduction}

The Acquired Immunodeficiency Syndrome (AIDS) as a major health threat to public health is the most important infectious pandemic of the late 20th and the early 21 st centuries across the globe. ${ }^{1-5}$

It is the fourth leading cause of death in the world, a major threat to low-income and poor countries, especially the Eastern Mediterranean countries such as Iran, since it can impose a large economic burden on countries. $^{5}$

In 2014, about 36.9 million people have been diagnosed to be infected with HIV. Also, 2 million new cases were diagnosed and 2.1 million deaths happened due to AIDS. In 2015, 2.1 million new cases of infection with HIV were reported. In 2016, 36.7 million people were infected with HIV and 1.8 million new cases of infection, and 1.0 million deaths were reported across the globe. ${ }^{5-8}$

According to national reports, the number of people living With HIV/AIDS (PLWHA) in Iran and the number of deaths were reported as 70,000 and 4,400, respectively. ${ }^{9}$ Also, 34,846 HIV-infected people have been identified in Iran until December 2016. However, it has been estimated that over 76,000 people are living with HIV in Iran. ${ }^{10}$

The age group of 15-24 years is recognized as the most vulnerable groups for HIV infection across the world, it has a significant effect on the reduction of life expectancy in countries with a high prevalence of the disease. Therefore, preventing, controlling, and caring for PLWHA is one of the most important activities of health care systems. ${ }^{2,11}$ Health care can reduce the new cases of diseases transmission in the

\footnotetext{
* Corresponding author. Social Determinants of Health Research Center, School of Public Health, Hamadan University of Medical Sciences, Hamadan, Iran.

E-mail address: u.mohammadi@umsha.ac.ir (Y. Mohammadi).
} 
community, can protect PLWHA from developing fatal diseases including cardiovascular disease (CVD) and liver problems. ${ }^{12}$ In 2016, the United Nations Program on HIV and AIDS (UNAIDS) as the $90-90-90$ program was launched. It suggested that by $2020,90 \%$ of people would be aware of their HIV status, $90 \%$ of those living with HIV would receive sustainable treatments, and $90 \%$ of those under the treatment would receive viral suppression. ${ }^{6,13}$

It has been estimated that only 70\% of PLWHA are aware of their health condition and to reach the goal of $90 \%$ diagnosis, 7.5 million people need access to HIV diagnostic testing services. Also, in mid2017, 20.9 million HIV infected people received Antiretroviral Therapy (ART). From 2000 to 2016, new HIV infections and AIDS-related deaths have fallen by $39 \%$ and one-third, respectively. Also, ART have saved the life of 13.1 million people as the result of extensive efforts using national HIV programs by the civil society and various development partners. ${ }^{5}$ Therefore, the access to and use of ART and health care services monitoring are prominent methods for increasing the survival rate and improving the quality of PLWHA. It can also help with the prevention of 21 million AIDS-related deaths and 28 million new infections by $2030.5,6,12,14$ Currently, $54 \%$ of adults and $43 \%$ of children with HIV are receiving ART treatment. The global coverage of ART for pregnant and lactating women has been reported to be $76 \%{ }^{5}$

The purpose of this study was to examine the health care utilization by PLWHA in Iran from 1987 to 2016. Data from this study can improve our understanding of resources used for health care and provide inputs to policymakers on HIV/AIDS health care services. It can be used to identify barriers to the use of health care services and help patients benefit from high quality services. Also, it can provide a basis for future studies in Iran.

\section{Methods}

\subsection{Data sources}

This study in an analytical descriptive study at the nation-wide. Samples were 30,516 HIV positive patients from 31 Iranian provinces registered in the Iranian health database from 1987 to October 2016. The data was collected from the Center for Communicable Diseases Control affiliated with the Iranian Ministry of Health and Medical Education (MOHME). Patient utilization from the use of health care services by HIV/AIDS patients for screening, preventing and curing health problems associated with AIDS. It included health care services including the Cluster of Differentiation 4 cell (CD4) count, Viral Load (VL) test, Purified Protein Derivation (PPD) test, tuberculosis (TB) evaluation, Antiretroviral (ARV), TB treatment, TB prophylaxis and Pneumocystis Carinii pneumonia (PCP) treatment.

\subsection{Statistical analysis}

The profile of health care service utilization were analyzed using descriptive and inferential statistics. To assess associated factors of the utilization services by patients in terms of age, gender, and marital status, Chi-square and Backward logistic regression tests were used and age is categorical into model. Also, the significance level less than 0.05 was considered statistically significant. The STATA v.11 software was used for the data analysis.

The study has been approved by the ethics Committee of Hamadan University of Medical Sciences. (NO. IR.UMSHA.RAC.1396.58).

\section{Results}

Out of 30516 HIV positive patients, $83.58 \%(n=25506)$ were male and $41.52 \%(\mathrm{n}=12670)$ were in the age group of $35-44$ years. Also, $35.24 \%$ of the patients were $(n=10754)$ single (Table 1$)$.

Table 2 showed health care utilization by PLWHA in Iran.

It was found that $45.18 \%$ and $4.13 \%$ of the patients performed CD4
Table 1

Demographic characteristics of patients with HIV/ AIDS in Iran.

\begin{tabular}{ll}
\hline Variable & $\mathrm{N}=30516$ \\
\cline { 2 - 2 } & $\mathrm{N} \mathrm{( \% )}$ \\
\hline Gender & \\
Female & $5009(16.41)$ \\
Male & $25506(83.58)$ \\
Age & \\
$0-4$ & $102(0.33)$ \\
$5-14$ & $388(1.27)$ \\
$15-24$ & $381(1.25)$ \\
$25-34$ & $4846(15.88)$ \\
$35-44$ & $12670(41.52)$ \\
$45-54$ & $6694(21.94)$ \\
55-64 & $2614(8.57)$ \\
$\geq 65$ & $2395(7.85)$ \\
Missed & $426(1.40)$ \\
Marriage Status & \\
Single & $10754(35.24)$ \\
Married & $10138(33.22)$ \\
Divorced & $3800(12.45)$ \\
Widowed & $1141(3.74)$ \\
Missed & $46683(15.35)$ \\
\hline
\end{tabular}

and VL tests, respectively. Also, 36.59\% of them received PPD test and $38.65 \%$ were screened for TB.

ARV was started by $33.71 \%$ of the patients and $27.76 \%$ of them received the latest ARV medication therapy. For TB treatment, $7.92 \%$ of the patients received the first prophylaxis of TB and $6.20 \%$ received the last TB prophylaxis. The last ARV treatment regime was discontinued in $6.33 \%$ of the patients, and $27.76 \%$ of them received the latest ARV medication. In $2.79 \%$ of the patients, the last TB treatment regime was discontinued. $1.47 \%$ of the patients had the last TB treatment regime and $5.38 \%$ of them discontinued the last prophylaxis of TB. Moreover, $16.76 \%$ of the patients received the first treatment for PCP, $13.44 \%$ received the latest PCP treatment. In 5.79\% of them PCP drug treatment was discontinued (Table 2).

Tables 3-5 showed the extent of the utilization of health care services by gender, age and the marital status. There was a statistically significant association between gender, age and the marital status, and receiving health care services. Overall, the probability of performing diagnostic tests such as CD4, VL and PPD, TB screening and treatment using ARV drugs, and PCP treatment was higher in females than in males (Table 3). It was also more in the younger age groups than higher age groups (Table 4), and by widows than singles (Table 5).

The time interval between the diagnosis of HIV/AIDS and receiving the first health care services by patients was reported in Table 6. Also, $1.10 \%$ of the patients were informed of the diagnosis on the same day, In $2.16 \%$ in the first week, $2.49 \%$ in the second week, $2.01 \%$ in the third week, and $1.45 \%$ in the fourth week after the diagnosis, they received health care services by health care centers (Table 6).

\section{Discussion}

This was the first and most comprehensive study in Iran to assess health care utilization by HIV/AIDS patients. The highest coverage of healthcare services was related to CD4 cells count, the evaluation of the latest status of TB, PPD diagnostic testing and treatment using ARV drugs. The coverage of health care services was very low (Table 2). Across the world, HIV-related stigma is considered the major international barrier to AIDS prevention and control, and PLWHA benefit from health care services. ${ }^{15,16}$ A study in Colombia (2012) found that HIVrelated stigma was an impediment to access to health care services by PLWHA. ${ }^{17}$

Stigma associated with HIV in patients has also been reported to be associated with a reduced use of healthcare service. In a study in Lima 
Table 2

Health care utilization status in HIV/AIDS patients.

\begin{tabular}{|c|c|}
\hline \multirow[t]{2}{*}{ Variable } & \multirow{2}{*}{$\begin{array}{l}\mathrm{N}=30516 \\
\mathrm{~N}(\%)\end{array}$} \\
\hline & \\
\hline \multicolumn{2}{|c|}{ First CD4 test } \\
\hline Yes & $13787(45.18)$ \\
\hline No & $16729(54.82)$ \\
\hline \multicolumn{2}{|c|}{ Last CD4 test } \\
\hline Yes & $13787(45.18)$ \\
\hline No & $16729(54.82)$ \\
\hline \multicolumn{2}{|c|}{ First VL test } \\
\hline Yes & $1260(4.13)$ \\
\hline No & $29256(95.87)$ \\
\hline \multicolumn{2}{|c|}{ Last VL test } \\
\hline Yes & $1260(4.13)$ \\
\hline NO & $29253(95.87)$ \\
\hline \multicolumn{2}{|c|}{ First PPD test } \\
\hline Yes & 1116 (36.59) \\
\hline No & $19349(63.41)$ \\
\hline \multicolumn{2}{|c|}{ Last PPD test } \\
\hline Yes & $11164(36.58)$ \\
\hline NO & $19352(63.42)$ \\
\hline \multicolumn{2}{|c|}{ latest evaluation of TB } \\
\hline Yes & $11795(38.65)$ \\
\hline No & $18721(61.35)$ \\
\hline \multicolumn{2}{|c|}{ start the first treatment of ARV } \\
\hline Yes & $10288(33.71)$ \\
\hline No & $20228(66.29)$ \\
\hline \multicolumn{2}{|c|}{ cut the last ARV regimen } \\
\hline Yes & $1931(6.33)$ \\
\hline No & $28585(93.67)$ \\
\hline \multicolumn{2}{|c|}{ last received ARV drug } \\
\hline Yes & $8470(27.76)$ \\
\hline No & $22046(72.24)$ \\
\hline \multicolumn{2}{|c|}{ cut the last TB regime } \\
\hline Yes & $851(2.79)$ \\
\hline No & $29665(97.21)$ \\
\hline \multicolumn{2}{|c|}{ Get the latest TB drug } \\
\hline Yes & $450(1.47)$ \\
\hline $\begin{array}{l}\text { No } \\
\text { start TB }\end{array}$ & 30066 (98.53) \\
\hline Yes & $2418(7.92)$ \\
\hline No & $28098(92.08)$ \\
\hline \multicolumn{2}{|c|}{ stop the latest TB prophylaxis } \\
\hline Yes & $1641(5.38)$ \\
\hline No & $28875(94.62)$ \\
\hline \multicolumn{2}{|c|}{ Get the latest TB prophylaxis } \\
\hline Yes & $1891(6.20)$ \\
\hline No & $28625(93.80)$ \\
\hline \multicolumn{2}{|c|}{ first PCP treatment } \\
\hline Yes & $5115(16.76)$ \\
\hline No & $25401(83.24)$ \\
\hline \multicolumn{2}{|c|}{ stop the latest PCP } \\
\hline Yes & 1766 (5.79) \\
\hline No & $28750(94.21)$ \\
\hline \multicolumn{2}{|c|}{ Get the latest PCP drug } \\
\hline Yes & $41100(13.44)$ \\
\hline No & 26416 \\
\hline
\end{tabular}

Peru, HIV-related stigma by healthcare providers had a negative effect on women's willingness to seek treatment. ${ }^{18}$ Also, a study in South Africa suggested that culture and context prevented the development of stigma in health care and also the use of related services by PLWHA. ${ }^{19}$ HIV-related stigma seems to worsen health care conditions for patients and can lead to the suppression of preventive measures, diagnostic tests, caring behaviors, more disease transmission, and can weaken the coverage of healthcare services for PLWHA. ${ }^{20}$ Therefore, governmental policies should aim at reducing discrimination against PLWHA to provide patients with a timely access to treatment and care. This study showed that women received more health care services than men (Table 3). Since women are important for the transmission of HIV/AIDS via sexual relationships or transmission to fetuses (18), receiving more services by this group is considered a strength point. The attitudes of health care workers (HCWs) also have a direct effect on receiving health care services. It has been shown that importance of the attitude, knowledge and behaviors of HCWs on the provision of health care services to patients, and patients willingness and ability to access care. ${ }^{12,21}$ Three studies in southern Italy, Nigeria and Taiwan on the assessment of the attitudes of HCWs towards serving PLWHA showed that providing education to enhance the knowledge of HCWs reduced their anxiety caused by exposure to the disease and increased the quality of health care services. ${ }^{12,22,23}$ Therefore, Planning for relevant continuing education of HIV/AIDS to all HCWs can have a significant impact on the improvement of patients' access to health care services.

In this study, $38.65 \%$ of the patients were evaluated for TB and $7.92 \%$ received TB prophylaxis (Table 2), which was not a suitable coverage. TB is the most common disease and the main cause of death among PLWHA. Also, the risk of TB for PLWHA reaches $8-10 \%$ per year, while the risk of TB infection for people without HIV infection in the lifetime is $5-10 \%$. Untreated TB accelerates the spread of HIV infection. ${ }^{9,24,25}$ A study in southeastern Ethiopia on 138 PLWHA over a two-year period (from January 2015 to December 30, 2016) showed that $36.9 \%$ ( 51 cases) of patients with TB and $72.5 \%$ (37 cases) of subjects had negative Results. ${ }^{26}$ Another study in India (2003) was conducted on 8640 PLWHA and showed that $93.5 \%$ of PLWHA were either co-infected over time with $\mathrm{TB}^{27}$ According to the WHO recommendations, TB screening should be carried out regularly at HIV surveillance centers. An early diagnosis of TB and treatment with antituberculosis and ARV drugs can prevent mortality from TB in PLWHA. ${ }^{5}$ One of the important signs for the success and progress of the HIV/AIDS care plan is an access to and benefiting from ATR drugs. An effective ARV regime can reduce the transmission of HIV and the HIV epidemic, and increase the survival and improvement of the quality of life of PLWHA. A study in South Africa (2014), confirmed that this result. ${ }^{28}$ Another study in Senegal on the long-term economic and social outcomes of the use of ARV drugs by PLWHA showed that socioeconomic indicators improved significantly since the onset of treatment. Also, medical care through ARV drugs helped limit the negative social impact of the disease. ${ }^{29}$

The Results of our study showed that only $33.71 \%$ of the patients received ARV drugs, 6.33\% discontinued the ARV regime, and $27.76 \%$ received the last ARV. Various factors can contribute to the continuous use of ARV drugs by the patient, including admission for the necessity of drug use up to the end of life, drug side effects, social issues, etc. The drugs are provided free of charge; therefore, it expected that the coverage of the ART drugs is higher than the results. The patients in younger age groups received more services than higher age groups (Table 4). Most patients were in the age group of 35-44 years, indicating that main victims of this disease were young people, ${ }^{2}$ The high coverage of younger age groups is considered a advantage. On the other, PLWHA are at the increased risk of developing cardiovascular diseases (CVD) and liver disorders. ${ }^{12}$ CVD and TB are common causes of death among PLWHA, ${ }^{25,30}$ and the risk of developing CVD is increased with age, based on a study on the assessment of the prevalence and incidence of CVD in Western Australia between 1995 and 2010. The majority of CVD occur in the old age. ${ }^{31}$ Another study on 150 patients with pulmonary TB in South Korea's Kyungpook showed that mortality of TB in older age groups was higher than younger age groups. ${ }^{32}$ Therefore, the high coverage of services in younger age groups can be seen as a disadvantage of caring for HIV, but all age groups should receive it. The risk of HIV has been linked to the marital status. A study on the relationship between the marital status and the risk of HIV infection in households in Nairobi found that the HIV prevalence among married men and women was $10.4 \%$ and $11.1 \%$, respectively and among divorced/separated/widowed men and women was $14.9 \%$ and $27.9 \%$, respectively. ${ }^{33}$ In a study on the relationship between the marital status and HIV-related mortality in the United States showed that divorced and separated individuals were 4.3 times more likely to die of HIV/AIDS than married individuals (adjusted relative risk (ARR): 
Table 3

Health care utilization of HIV/AIDS patients by gender.

\begin{tabular}{|c|c|c|c|c|}
\hline \multirow{2}{*}{$\begin{array}{l}\text { Gender } \\
{ }^{\mathrm{a}} \text { Female }\end{array}$} & \multicolumn{4}{|l|}{$\mathrm{N}=30516$} \\
\hline & OR Crude (95\% CI) & $P$-value & OR Adjust $(95 \% \mathrm{CI})$ & $P$-value \\
\hline First CD4 test & $0.34(0.32,0.36)$ & $<0.001$ & $0.31(0.29,0.33)$ & $<0.001$ \\
\hline Last CD4 test & $0.34(0.32,0.36)$ & $<0.001$ & $0.31(0.29,0.33)$ & $<0.001$ \\
\hline First VL test & $0.34(0.30,0.39)$ & $<0.001$ & $0.35(0.31,0.40)$ & $<0.001$ \\
\hline Last VL test & $0.34(0.30,0.39)$ & $<0.001$ & $0.35(0.31,0.40)$ & $<0.001$ \\
\hline First PPD test & $0.44(0.41,0.47)$ & $<0.001$ & $0.40(0.38,0.43)$ & $<0.001$ \\
\hline Last PPD test & $0.44(0.41,0.47)$ & $<0.001$ & $0.40(0.38,0.43)$ & $<0.001$ \\
\hline latest evaluation of TB & $0.40(0.38,0.42)$ & $<0.001$ & $0.38(0.36,0.41)$ & $<0.001$ \\
\hline start the first treatment of ARV & $0.30(0.28,0.32)$ & $<0.001$ & $0.28(0.27,0.30)$ & $<0.001$ \\
\hline cut the last ARV regimen & $0.91(0.80,1.03)$ & 0.143 & $0.85(0.75,0.96)$ & $<0.001$ \\
\hline last received ARV drug & $0.34(0.32,0.36)$ & $<0.001$ & $0.34(0.31,0.36)$ & 0.012 \\
\hline cut the last TB regime & $2.54(1.96,3.29)$ & $<0.001$ & $2.26(1.74,2.94)$ & $<0.001$ \\
\hline Get the latest TB drug & $1.51(1.12,2.01)$ & 0.005 & $1.37(1.02,1.85)$ & 0.033 \\
\hline start TB prophylaxis & $0.91(0.82,1.01)$ & 0.134 & $0.85(0.76,0.95)$ & 0.007 \\
\hline stop the latest TB prophylaxis & $1.11(0.97,1.28)$ & 0.111 & $1.03(0.90,1.19)$ & 0.594 \\
\hline Get the latest TB prophylaxis & $0.79(0.70,0.89)$ & $<0.001$ & $0.75(0.66,0.85)$ & $<0.001$ \\
\hline first PCP treatment & $0.68(0.63,0.73)$ & $<0.001$ & $0.63(0.58,0.68)$ & $<0.001$ \\
\hline stop the latest PCP & $0.67(0.59,0.75)$ & $<0.001$ & $0.62(0.55,0.70)$ & $<0.001$ \\
\hline Get the latest PCP drug & $0.69(0.63,0.75)$ & $<0.001$ & $0.65(0.60,0.71)$ & $<0.001$ \\
\hline
\end{tabular}

a Reference group.

4.321, 95\% confidence interval (CI): $2.978,6.269$ ). Single/never married persons were 13 times as likely to die of HIV/AIDS as their married counterparts (ARR: 13.092, 95\% CI: 9.652, 17.757). ${ }^{34}$

In this study, the chance of receiving health care services by widows was significantly higher than singles (Table 5). On the other, they mostly were single (35.24\%). Evidence on the relationship between the marital status and the risk of HIV-related deaths is controversial and depends on various demographic and sexual behaviors. For those who are married, there is a need for more preventive strategies based on social and cultural conditions. ${ }^{35}$ Most patients have been single indicating the presence of high-risk behaviors related to HIV/AIDS in this group.

Therefore, a poor coverage of receiving health care services by this group is a weakness. $1.10 \%$ of the patients on the first day, $1.45 \%$ in the first month and $39.03 \%$ in the first five years of the diagnosis, received the first health care services (Table 6). Given that the time interval between the diagnosis and receiving health care by a patient influences her/his quality of life and survival rate, effective screening, especially in high-risk locations such as prisons and among homeless people should be ensured. This study showed serious weaknesses in the coverage of health care services provided to PLWHA. The services provided under the national protocol by healthcare system have been improved over the years, but the service coverage remains poor. Provision of injecting drug facilities, educational and occupational status, disabilities and illnesses, distance from the workplace or home from the service provider have not been evaluated in this study. Another limitation of this study was that the registration system of the disease in Iran has improved over years, and information of the number of patients identified in recent years is lacking. It can reduce the validity of estimates of patient benefits from national health care services. It has been estimated that over 76,000 people are living with HIV in Iran, most of whom are not registered, and given that we have used the recorded data, our Results may be problematic for generalization. ${ }^{10}$ Planning for a proper supplying and distribution of HCWs and specialized facilities, drug provision, continuous education of HCWs, continuous monitoring and evaluation of HIV service providers, improvement of the registration system through the use of experienced and educated staff, and continuous monitoring of the registration system

Table 4

Health care utilization of HIV/AIDS patients by age.

\begin{tabular}{|c|c|c|c|c|}
\hline & $\mathrm{N}=30516$ & & & \\
\hline First CD4 test & $0.66(0.65,0.68)$ & $<0.001$ & $0.80(0.87,0.82)$ & $<0.001$ \\
\hline First VL test & $0.72(0.69,0.76)$ & $<0.001$ & $0.85(0.80,0.90)$ & $<0.001$ \\
\hline Last VL test & $0.72(0.69,0.76)$ & $<0.001$ & $0.85(0.80,0.90)$ & $<0.001$ \\
\hline First PPD test & $0.74(0.73,0.76)$ & $<0.001$ & $0.89(0.87,0.92)$ & $<0.001$ \\
\hline Last PPD test & $0.74(0.73,0.76)$ & $<0.001$ & $0.89(0.87,0.92)$ & $<0.001$ \\
\hline cut the last ARV regimen & $0.96(0.93,1.00)$ & 0.092 & $1.14(1.09,1.20)$ & $<0.001$ \\
\hline last received ARV drug & $0.72(0.70,0.73)$ & $<0.001$ & $0.84(0.82,0.86)$ & $<0.001$ \\
\hline cut the last $\mathrm{TB}$ regime & $1.03(0.97,1.09)$ & 0.227 & $1.23(1.15,1.32)$ & $<0.001$ \\
\hline Get the latest TB drug & $0.91(0.84,0.98)$ & 0.022 & $1.01(0.92<1.11)$ & 0.740 \\
\hline start TB prophylaxis & $0.95(0.92,0.99)$ & 0.012 & $1.12(1.07,1.17)$ & $<0.001$ \\
\hline stop the latest TB prophylaxis & $1.04(1.00,1.09)$ & 0.023 & $1.26(1.20,1.32)$ & $<0.001$ \\
\hline Get the latest $\mathrm{TB}$ prophylaxis & $0.88(0.85,0.92)$ & $<0.001$ & $1.00(0.96,1.05)$ & 0.739 \\
\hline
\end{tabular}

${ }^{\text {a }}$ Reference group. 
Table 5

Health care utilization of HIV/AIDS patients by marriage Status.

\begin{tabular}{|c|c|c|c|c|}
\hline \multirow{2}{*}{$\begin{array}{l}\text { Marriage status } \\
{ }^{\mathrm{a}} \text { Single }\end{array}$} & \multicolumn{4}{|l|}{$\mathrm{N}=30516$} \\
\hline & OR crude $(95 \% \mathrm{CI})$ & $P$-value & OR adjust $(95 \% \mathrm{CI})$ & $P$-value \\
\hline First CD4 test & $1.24(1.21,1.28)$ & $<0.001$ & $1.18(1.14,1.22)$ & $<0.001$ \\
\hline Last CD4 test & $1.24(1.21,1.28)$ & $<0.001$ & $1.18(1.14,1.22)$ & $<0.001$ \\
\hline First VL test & $1.10(1.03,1.18)$ & 0.002 & $1.00(0.93,1.08)$ & 0.835 \\
\hline Last VL test & $1.10(1.03,1.18)$ & 0.002 & $1.00(0.93,1.08)$ & 0.835 \\
\hline First PPD test & $1.21(1.18,1.25)$ & $<0.001$ & $1.13(1.10,1.17)$ & $<0.001$ \\
\hline Last PPD test & $1.21(1.18,1.25)$ & $<0.001$ & $1.13(1.10,1.17)$ & $<0.001$ \\
\hline latest evaluation of $\mathrm{TB}$ & $1.23(1.20,1.27)$ & $<0.001$ & $1.19(1.15,1.23)$ & $<0.001$ \\
\hline start the first treatment of ARV & $1.28(1.24,1.32)$ & $<0.001$ & $1.18(1.14,1.22)$ & $<0.001$ \\
\hline cut the last ARV regimen & $1.09(1.03,1.15)$ & 0.001 & $1.11(1.05,1.18)$ & $<0.001$ \\
\hline last received ARV drug & $1.25(1.22,1.29)$ & $<0.001$ & $1.17(1.13,1.21)$ & $<0.001$ \\
\hline cut the last TB regime & $0.84(0.77,0.92)$ & $<0.001$ & $0.95(0.86,1.03)$ & 0.263 \\
\hline Get the latest TB drug & $0.99(0.88,1.11)$ & 0.903 & $1.07(0.95,1.21)$ & 0.205 \\
\hline start TB prophylaxis & $1.12(1.06,1.17)$ & $<0.001$ & $1.14(1.09,1.20)$ & $<0.001$ \\
\hline stop the latest TB prophylaxis & $1.10(1.04,1.17)$ & 0.001 & $1.16(1.09,1.23)$ & $<0.001$ \\
\hline Get the latest TB prophylaxis & $1.13(1.07,1.19)$ & $<0.001$ & $1.14(1.07,1.20)$ & $<0.001$ \\
\hline first PCP treatment & $1.13(1.09,1.17)$ & $<0.001$ & $1.11(1.07,1.15)$ & $<0.001$ \\
\hline stop the latest PCP & $1.14(1.07,1.20)$ & $<0.001$ & $1.11(1.04,1.18)$ & $<0.001$ \\
\hline Get the latest PCP drug & $1.12(1.08,1.16)$ & $<0.001$ & $1.11(1.06,1.15)$ & $<0.001$ \\
\hline
\end{tabular}

\section{a Reference group.}

Table 6

The time interval between the diagnosis of the disease and receiving the first service by HIV patients.

\begin{tabular}{ll}
\hline Time & N (\%) \\
\hline Day/Week & \\
First day & $335(1.10)$ \\
First week & $659(2.16)$ \\
Second week & $759(2.49)$ \\
Third week & $613(2.01)$ \\
Forth week & $444(1.45)$ \\
Year & \\
$0-5$ & $11910(39.03)$ \\
$6-10$ & $1583(5.19)$ \\
$11-15$ & $268(0.88)$ \\
$16-20$ & $19(0.06)$ \\
$21-25$ & $6(0.02)$ \\
$26-28$ & $1(0.00)$ \\
Missed & $16729(54.82)$ \\
\end{tabular}

can improve the use of health care services. In health care systems, HCWs should receive more education and follow up patients, and health care service centers should be located in places that are not publicly displayed to provide better referrals of PLWHA.

\section{Conclusions}

While the process of providing health care services to such patients has improved in recent years, it is far from the expected level and the overall coverage is very low. Planning for the appropriate supply and distribution of Health Care Workers (HCWs) and specialized facilities, provision of drugs, continuous education of HCWs, improvement of registration and reporting of HIV/AIDS can enhance the provision of healthcare services to these patients.

\section{Funding}

The study was funded by the Vice-chancellor for Research and Technology, Hamadan University of Medical Sciences (No. 960115280).

\section{Conflicts of interest}

The authors declare that they have no conflict of interest.

\section{Acknowledgements}

We would like to thank staff of Iranian Ministry of Health, especially Dr. Akbapour for technical help.

\section{References}

1. Pendse R, Gupta S, Yu D, Sarkar S. HIV/AIDS in the South-East Asia region: progress and challenges. $J$ Virus Erad. 2016;2(Suppl 4):1.

2. Elbadawi A, Mirghani H. Depression among HIV/AIDS Sudanese patients: a crosssectional analytic study. Pan Afr Med J. 2017;26.

3. Trapero-Bertran M, Oliva-Moreno J. Economic impact of HIV/AIDS: a systematic review in five European countries. Health Econ Rev. 2014;4(1):15.

4. Shahbazi M, Farnia M, Rahmani K, Moradi G. Trend of HIV/AIDS prevalence and related interventions administered in prisons of Iran-13 years' experience. Iran $J$ Public Health. 2014;43(4):471.

5. Chan M. HIV/AIDS. WHO; 2018. Available from: http://www.who.int/news-room/ fact-sheets/detail/hiv-aids, Accessed date: 15 February 2018.

6. Foresto JS, Melo ES, Costa CRB, Antonini M, Gir E, Reis RK. Adherence to antiretroviral therapy by people living with HIV/AIDS in a municipality of São Paulo. Rev Gaucha Enferm. 2017;38(1).

7. Ertunc B, Kaya S, Koksal I. Clinico-epidemiological analysis of HIV/AIDS patients. Eurasian J Med. 2016;48(3):157.

8. Abu EK, Abokyi S, Obiri-Yeboah D, et al. Retinal microvasculopathy is common in HIV/AIDS patients: a cross-sectional study at the Cape Coast Teaching Hospital, Ghana. J Ophthalmol. 2016;2016.

9. Doosti-Irani A, Holakouie-Naieni K. Determination the Research priorities in the field of HIV/AIDS in Iran: a systematic review article. Iran $J$ Public Health. 2016;45(9):1149

10. Amiri FB, Doosti-Irani A, Sedaghat A, Fahimfar N, Mostafavi E. Knowledge, attitude, and practices regarding HIV and TB among homeless people in Tehran, Iran. Int $J$ Health Pol Manag. 2018;7(6):549.

11. Anwuri GC, Dunn MS, Schulze F. Determinants of voluntary HIV/AIDS counseling and testing among community college students in the United States. Int J MCH AIDS. 2017;6(2):109.

12. Ledda C, Cicciù F, Puglisi B, Ramaci T, Nunnari G, Rapisarda V. Attitude of health care workers (HCWs) toward patients affected by HIV/AIDS and drug users: a crosssectional study. Int J Environ Res Publ Health. 2017;14(3):284.

13. Das S, Opoku J, Kharfen M, Allston A. Geographic patterns of poor HIV/AIDS care continuum in District of Columbia. AIDS Res Ther. 2018;15(1):2.

14. Hipolito RL, Oliveira DCd, Costa TLd, Marques SC, Pereira ER, Gomes AMT. Quality of life of people living with HIV/AIDS: temporal, socio-demographic and perceived health relationship. Rev Latino-Am Enferm. 2017;25.

15. Eaton LA, Earnshaw VA, Maksut JL, Thorson KR, Watson RJ, Bauermeister JA. Experiences of stigma and health care engagement among Black MSM newly diagnosed with HIV/STI. J Behav Med. 2018:1-9.

16. Wagner AC, Hart TA, McShane KE, Margolese S, Girard TA. Health care provider attitudes and beliefs about people living with HIV: initial validation of the health care provider HIV/AIDS Stigma Scale (HPASS). AIDS Behav. 2014;18(12):2397-2408.

17. Bermúdez-Román V, Bran-Piedrahita L, Palacios-Moya L, Posada-Zapata IC. Influence of HIV related stigma on health care access. Salud Publica Mex. 2015;57(3):252-259. 
18. Valencia-Garcia D, Rao D, Strick L, Simoni JM. Women's experiences with HIV-related stigma from health care providers in Lima, Peru:"I would rather die than go back for care". Health Care Women Int. 2017;38(2):144-158.

19. Okoror TA, BeLue R, Zungu N, Adam AM, Airhihenbuwa CO. HIV positive women's perceptions of stigma in health care settings in Western Cape, South Africa. Health Care Women Int. 2014;35(1):27-49.

20. Wu S, Li L, Wu Z, et al. A brief HIV stigma reduction intervention for service providers in China. AIDS Patient Care STDS. 2008;22(6):513-520.

21. Onadeko MO, Balogun MO, Onigbogi OO, Omokhodion FO. Occupational exposure, attitude to HIV-positive patients and uptake of HIV counselling and testing among health care workers in a tertiary hospital in Nigeria. SAHARA-J: J Soc Aspects HIV/ AIDS. 2017;14(1):193-201.

22. Sadah A, Fawole A, Sadoh W, Oladimeji A, Sotiloye O. Attitude of health-care workers to HIV/AIDS. Afr J Reprod Health. 2006;10(1):39-46.

23. See L-C, Shen Y-M, Chen C-L, et al. Professional attitude of health care workers toward serving HIV/AIDS patients and drug users: questionnaire design and evaluation of reliability and validity. AIDS Care. 2011;23(11):1448-1455.

24. Phetlhu DR, Bimerew M, Marie-Modeste RR, Naidoo M, Igumbor J. Nurses' knowledge of tuberculosis, HIV, and integrated HIV/TB care policies in rural Western Cape, South Africa. J Assoc Nurses AIDS Care. 2018:29(6):876-886.

25. Pathmanathan I, Ahmedov S, Pevzner E, et al. TB preventive therapy for people living with HIV: key considerations for scale-up in resource-limited settings. Int $J$ Tubercul Lung Dis. 2018;22(6):596-605.

26. Negussie A, Debalke D, Belachew T, Tadesse F. Tuberculosis co-infection and its associated factors among People living with HIV/AIDS attending antiretroviral therapy clinic in southern Ethiopia: a facility based retrospective study. BMC Res Notes. 2018;11(1):417.

27. Maniar JK, Kamath RR, Mandalia S, Shah K, Maniar A. HIV and tuberculosis: partners in crime. Indian J Dermatol, Venereol Leprol. 2006;72(4):276.

28. Mastro TD, Sista N, Abdool-Karim Q. ARV-based HIV prevention for women-where we are in 2014. J Int AIDS Soc. 2014:17:19154.

29. Coutherut J. Socio-economic impact of one decade of ARV therapy for people living with HIV in Senegal. Bull Soc Pathol Exot (1990). 2014;107(4):261-265.

30. Laurence J, Elhadad S, Ahamed J. HIV-associated cardiovascular disease: importance of platelet activation and cardiac fibrosis in the setting of specific antiretroviral therapies. Open Heart. 2018;5(2):e000823.

31. Sarink D, Nedkoff L, Briffa T, et al. Trends in age-and sex-specific prevalence and incidence of cardiovascular disease in Western Australia. Eur J Prev Cardiol. 2018;25:1280-1290.

32. Lee J, Lim JK, Kim EJ, et al. Comparison of clinical manifestations and treatment outcome according to age groups in adult patients with miliary tuberculosis. $J$ Thorac Dis. 2018;10(5):2881-2889.

33. Kimani JK, Ettarh R, Ziraba AK, Yatich N. Marital status and risk of HIV infection in slum settlements of Nairobi, Kenya: Results from a cross-sectional survey. Afr $J$ Reprod Health. 2013;17(1):103-113.

34. Kposowa AJ. Marital status and HIV/AIDS mortality: evidence from the US national longitudinal mortality study. Int J Infect Dis. 2013;17(10):e868-e874.

35. Shisana O, Zungu-Dirwayi N, Toefy Y, Simbayi L, Malik S, Zuma K. Marital status and risk of HIV infection in South Africa. S Afr Med J. 2004;94(7):537-543. 